\title{
CARACTERÍSTICAS LINGÜÍSTICAS QUE AFECTAN LA ADQUISICIÓN DE LA MORFOLOGÍA DERIVATIVA NOMINAL EN INGLÉS COMO L2
}

\section{LINGUISTIC CHARACTERISTICS THAT AFFECT THE ACQUISITION OF NOMINAL DERIVATIONAL MORPHOLOGY IN ESL}

\author{
Brenda Vargas Vega \\ Eva P. Velásquez Upegui \\ Universidad Autónoma de Querétaro \\ brenda.vargas@uaq.mx, evapvelasquez@gmail.com
}

Enviado: 01/06/2018

Aceptado: 05/10/2018

\begin{abstract}
Resumen
Los estudios morfológicos de adquisición de L2 han evaluado principalmente el conocimiento sintáctico de los morfemas derivativos. En este artículo se presenta una propuesta para evaluar el conocimiento distribucional de algunos nominalizadores deverbales con el objetivo de determinar qué características morfológicas y léxicas tienen mayor incidencia en su aprendizaje cuando estos sufijos generan el valor semántico de resultado. Las variables a estudiar fueron el nivel de dificultad y la productividad del sufijo; así como la propiedad de cognado y la frecuencia de las palabras que generan. Los datos provienen de una modificación a la prueba de derivación de Carlisle (2000) aplicada a 130 universitarios mexicanos. Los resultados muestran que las cuatro características tienen un efecto significativo en la adquisición, aunque
\end{abstract}

\begin{abstract}
The morphological studies of second language acquisition have concentrated primarily on the syntactic knowledge of derivational morphemes. This study presents a proposal to evaluate the distributional knowledge of some deverbal nominalizers to determine what morphological and lexical characteristics affect learning when these suffixes create a resultative semantic value. Four variables were analyzed: the level of difficulty and productivity of the suffix; and the cognate status and frequency of the derived words. The data comes from a modified version of the derivational test of Carlisle (2000) applied to130 Mexican university students. The results show a significant statistical effect of the four variables, indicating that the level of difficulty of the morpheme has the greatest influence
\end{abstract}

Para citar este artículo / To cite this article: Vargas Vega, Brenda y Eva P. Velásquez Upegui (2018). Características lingüísticas que afectan la adquisición de la morfología derivativa nominal en inglés como L2. ELUA, 32: 349-365. doi: 10.14198/ELUA2018.32.16

Enlace / Link: http://dx.doi.org/10.14198/ELUA2018.32.16 
el bajo nivel de dificultad del morfema es la característica que más facilita el aprendizaje de los nominalizadores para hablantes nativos de español, presentando implicaciones pedagógicas y metodológicas.

PALABRAS CLAVE: Morfología derivativa, conocimiento distribucional, nominalizadores deverbales, inglés como L2. on morphological learning for Spanish native speakers. The study presents pedagogical and methodological implications.

KEYWORDS: derivational morphology, distributional knowledge, deverbal nominalizers, ESL.

\section{INTRODUCCIÓN}

Un área de interés de la adquisición y enseñanza del inglés como L2 es la morfología porque según diversos autores, ésta incide en el conocimiento léxico y la comprensión lectora en una segunda lengua (Kieffer y Lesaux, 2007, 2008 y 2012; Ramírez et alii., 2010 y 2011; Curinga, 2013; Varatharajoo et alii., 2015). El estudio de la morfología derivativa, sin embargo, se ha centrado más en el conocimiento relacional; i.e. determinar si dos palabras están relacionadas; o bien, en el conocimiento sintáctico - identificar que los sufijos derivativos marcan la categoría sintáctica de las palabras en inglés, dejando de lado el conocimiento distribucional, que se refiere al conocimiento de las restricciones de selección en la concatenación de bases y afijos. Tyler y Nagy (1989) hacen estas distinciones de los diferentes aspectos del conocimiento morfológico y afirman que el conocimiento distribucional es el último en el proceso de adquisición morfológica derivativa del inglés como L1 pero desconocemos si esto mismo sucede en la adquisición del inglés como L2.

En el estudio de la adquisición morfológica de la L2, destacamos la contribución de Bauer y Nation (1993), quienes considerando la frecuencia, regularidad, productividad y predictibilidad de los sufijos flexivos y derivativos, proponen un conjunto graduado de siete niveles que, a su parecer, puede ser útil para la enseñanza de inglés como L2 ${ }^{1}$. Estos niveles han sido usados por varios investigadores de inglés como L2 para dar cuenta del orden de adquisición de los afijos con participantes de diferente L1 y su relación con el conocimiento léxico (Mochizuki y Aizawa, 2000, Medellín y Auza, 2008; Danilović et alii., 2013) así como para estudiar el conocimiento relacional de los afijos (Schmitt y Zimmerman, 2002; Ward y Chuenjundaeng, 2009).

El presente trabajo es parte de un proyecto doctoral en el que se estudia la morfología derivativa nominal en el proceso de adquisición del inglés como L2, enfocándonos en cuatro nominalizadores deverbales que generan el valor semántico de resultado: -ation (ejem: separate -> separation), -ment (ejem: arrange -> arrangement), -al (ejem: arrive -> arrival), $-y$ (ejem: deliver $->$ delivery). Nuestros principales objetivos son determinar qué propiedad de los morfemas tiene mayor incidencia en el aprendizaje de los nominalizadores deverbales: su productividad o su nivel de dificultad, e identificar si el aprendizaje de algunos nominalizadores deverbales del inglés se facilita por la alta frecuencia de los sustantivos que generan, o la propiedad de cognado de las bases a la que se añaden. Esta investigación contribuye al estudio de nominalizadores deverbales y a la morfología derivativa en general al analizar cuáles características de los morfemas y de las palabras facilitan la adquisición

1 Los afijos correspondientes a cada nivel pueden consultarse en Bauer, L., y Nation, P. (1993). Ver también nota al pie 3 . 
de los sufijos en los casos donde hay empalme de función y significado. El estudio es pertinente debido a que hay pocas investigaciones que aborden el problema del conocimiento distribucional de los morfemas, aun cuando propicia confusión en los estudiantes. En este artículo se presentarán los resultados cuantitativos de dicho estudio.

La pregunta de investigación que nos proponemos responder es: ¿Qué factores lingüísticos asociados al morfema y a la palabra tienen mayor incidencia en el aprendizaje de la morfología derivativa nominal inglesa para hablantes de español como lengua materna? Partimos de la hipótesis de que los sufijos y las palabras derivadas tienen características que facilitan su adquisición. Las variables morfológicas a estudiar fueron el nivel de dificultad (Bauer y Nation, 1993) y la productividad del sufijo con la función de nominalización (calculada a partir del corpus Morphoquantics) ${ }^{2}$. Las variables léxicas que se seleccionaron para el estudio fueron la frecuencia del sustantivo deverbal (como aparece en el corpus Morphoquantics) y la propiedad de cognado de los verbos de donde se generan estos sustantivos. El artículo comienza con el marco teórico, seguido por la descripción detallada de la elaboración del instrumento, para luego mostrar la incidencia de las características morfológicas y léxicas en la adquisición de los morfemas. Finalmente, veremos la discusión y las conclusiones.

\section{MARCO TEÓRICO}

Esta sección describe los estudios que se usaron como base teórica o metodológica para realizar este trabajo de investigación. Comienza describiendo lo que Bauer y Nation (1993) entienden por las propiedades de frecuencia, regularidad, productividad y predictibilidad ya que basados en ellas, proponen un conjunto graduado de afijos con diferente nivel de dificultad. Después se explica la diferencia entre los aspectos relacionales, sintácticos y distribucionales de la morfología derivativa propuestos por Tyler y Nagy (1989) y finalmente se exponen algunas variables que pueden afectar la adquisición morfológica.

Bauer y Nation (1993) definen frecuencia como el número de palabras en las que ocurre un afijo y productividad como la probabilidad de que el afijo se use para formar nuevas palabras. Si ambas son altas, el afijo es más fácil de adquirir ya que el hablante o aprendiente tendrá más exposición a él. La regularidad en la forma escrita y oral de la base del afijo y de la función del afijo también facilitan la adquisición. Es decir, el hecho de que la base no sufra modificaciones ortográficas y/o fonológicas cuando se le añade un afijo y que el afijo no tenga alomorfos ni múltiples funciones gramaticales lo hacen más fácil de adquirir. Por último, la predictibilidad se refiere a que el significado del afijo a veces no se sabe hasta que se ve la base a la que se añade como el significado del sufijo $-s$, que puede indicar plural o la marca de la tercera persona singular en contraste a -ed que sólo marca la forma regular del pasado. Los autores sugieren que una mayor predictibilidad del sufijo facilita la adquisición.

De acuerdo a Tyler y Nagy (1989), se pueden distinguir diferentes aspectos del conocimiento de la morfología derivativa que se desarrollan en diferentes momentos de la adquisición. El primer aspecto es el conocimiento relacional y se refiere al conocimiento

2 El corpus será descrito en la sección 2; por ahora solo cabe resaltar que un sufijo puede tener más de una función gramatical y/o valor semántico. Por ejemplo, el sufijo -al puede generar nombres (ejem: propose -> proposal) o adjetivos (ejem: accident -> accidental). Asimismo, el sufijo -an puede generar gentilicios (Russian), denotar afiliación o creencia (Republican, Christian) o ser relativo a algo (communitarian, vegetarian). 
o percepción de que dos palabras están relacionadas como argue-argument en oposición a off-offer. Al segundo aspecto se le conoce como conocimiento sintáctico y consiste en saber que los sufijos derivativos marcan la categoría sintáctica de las palabras en inglés (ejem: el sufijo -ize genera verbos mientras el sufijo -(a)tion forma sustantivos). Se piensa que el sufijo derivativo provee información confiable acerca de la categoría sintáctica de la palabra aun cuando uno no sepa el significado de la base, dejándonos saber que ambiguity es un sustantivo y ambiguous es un adjetivo aun sin saber el significado de ambigu-. Por último, el conocimiento distribucional es el conocimiento de las restricciones en la concatenación de bases y afijos. Por ejemplo, en el caso de los nominalizadores, el sufijo -ness se añade a adjetivos y a sustantivos pero no a verbos (quietness, childness vs * playness). Tyler y Nagy (1989) afirman que este aspecto es el último en la adquisición del inglés como L1 y es el tipo de conocimiento que se evalúa en este trabajo debido a que existen muchos trabajos que evalúan el conocimiento relacional y sintáctico de los morfemas en inglés como L2 (Mochizuki y Aizawa, 2000; Schmitt y Zimmerman, 2002; Ku y Anderson, 2003; Lardiere, 2006; Medellín y Auza, 2008; Ward y Chuenjundaeng, 2009; Khodadoust et alii., 2013; Danilović et alii., 2013; Varatharajoo et alii., 2015) pero hace falta investigar la adquisición del aspecto distribucional.

Entre las variables léxicas que pueden afectar la adquisición morfológica se encuentran los cognados, la frecuencia de la base o de la palabra derivada, la longitud de palabra y la transparencia ortográfica, fonológica y/o semántica de los derivados ${ }^{3}$. Entre las variables morfológicas destacan la neutralidad de $\operatorname{los}_{\text {afijos }}{ }^{4}$, su productividad ${ }^{5}$, su plurifuncionalidad, su alomorfia y su polisemia. Además de variables lingüísticas, también se deben considerar variables personales (L1, nivel de la L2, etc.) y tomar decisiones sobre el diseño de las pruebas (qué tipo de conocimiento se pretende medir, si se incluyen pseudo-palabras o no, si es una tarea de comprensión o de producción, etc.). En este estudio se controlaron las variables léxicas de cognado y frecuencia; las propiedades morfológicas de productividad y dificultad y se aplicó una prueba de producción controlada (completar oraciones) a hablantes nativos de español con diferente nivel de inglés como L2. Algunas investigaciones que han estudiado estas variables sugieren lo siguiente:

3 La transparencia es una cualidad de las palabras derivadas que puede facilitar su adquisición. Las palabras derivadas que son ortográfica y fonológicamente transparentes (Carlise, 2000) son aquellas que no sufren modificaciones en estos aspectos cuando se añaden los afijos a sus bases, permitiéndoles conservar su forma completa (move->movement, power->powerful) en oposición a las palabras no transparentes, que sufren cambios ortográficos y/o fonológicos (nature->natural, easy-> easily). Esta característica está ligada a la neutralidad de los sufijos. Las palabras derivadas también pueden ser semánticamente transparentes; es decir, que se puede inferir su significado de manera composicional como en teach (enseñar) -> teacher (alguien que enseña) o semánticamente opacas como depart (salir) -> department (que no es la acción o resultado de salir sino departamento, entendido como la división de una organización como el departamento de ventas) (Marslen-Wilson et.alii., 1994).

4 De acuerdo a Tyler y Nagy (1989), los sufijos neutrales difieren de los no neutrales en que los primeros se añaden a morfemas libres de modo que cuando se eliminan, el resultado es una palabra independiente (own - owner) y no causan cambios vocálicos o de estrés en la base a la que se añaden. Los autores puntualizan que los sufijos neutrales usualmente generan palabras derivadas con transparencia semántica y que este tipo de sufijos tiene una gran aplicabilidad; por ejemplo, el sufijo - er puede añadirse a casi cualquier verbo para formar un agentivo. En contraste, los morfemas no neutrales no cuentan con estas características facilitadoras. Por ejemplo, la raíz fer de prefer y refer toma el sufijo nominalizador -ence y éste ocasiona cambios fonológicos (pre-fér -> pré-fer -ence, re-fér $->$ ré-fer-ence).

5 Se dice que un proceso morfológico es productivo si puede usarse sincrónicamente en la creación de nuevas palabras (Bauer, 1983:18). 
o Los participantes suelen obtener mejores resultados en las tareas de comprensión que en las de producción morfológica (Khodadoust et alii., 2013; Alotaibi y Alotaibi, 2017).

o Existe un efecto de la L1 en pruebas morfológicas de la L2 (Lowie, 2000 y Jiang et.alii., 2011)

o Los cognados y la alta frecuencia facilitan el aprendizaje de una L2 (Costa et alii., 2000; Bravo et alii., 2007; Kelley y Kohnert, 2012 y Peeters et alii., 2013).

o Los morfemas de alta productividad se adquieren más temprano que los de baja productividad (Lowie, 2005).

o La baja dificultad de los morfemas puede facilitar su aprendizaje (Bauer y Nation, 1993).

o Las variables no suelen trabajar de manera aislada, sino en interacción (Bertram et alii., 2000; Curinga, 2013; Alotaibi y Alotaibi, 2017)

Basados en estos hallazgos, esperamos que las palabras derivadas que sean cognadas, de alta frecuencia y que incluyan sufijos de alta productividad y baja dificultad obtengan mejores resultados que las palabras derivadas que carezcan de estas características.

\section{UNA PROPUESTA PARA ESTUDIAR EL CONOCIMIENTO DISTRIBUCIO- NAL DE LOS MORFEMAS DERIVATIVOS CON LOS SUFIJOS NOMINALI- ZADORES - ATION, -MENT, -AL, -Y}

Como se dijo anteriormente, la mayoría de las investigaciones sobre morfología derivativa estudian el conocimiento sintáctico de los sufijos; i.e. evalúan si los participantes reconocen la clase gramatical de las palabras derivadas que generan los sufijos. Sin embargo, para evaluar el conocimiento distribucional es necesario elegir sufijos que cumplan la misma función gramatical; y para evitar que el contenido semántico del derivado afecte la adquisición, es necesario que dichos sufijos tengan el mismo significado. Bajo estas condiciones, se elaboró una prueba morfológica que contiene seis sufijos que se añaden a verbos: cuatro de estos sufijos se consideraron experimentales por tener la misma función gramatical (nominalización deverbal) y porque todos generan el significado de acción o resultado; y los dos sufijos restantes son distractores. Mientras uno se distingue por su función gramatical al crear adjetivos a partir de verbos (observe-> observable), el otro se distingue en su valor semántico, al generar nombres con significado de agente (compose->composer). En este artículo únicamente se presentan los resultados de los sufijos experimentales.

En los siguientes párrafos describiremos cómo se hizo la elección de los sufijos (sección 3.1) y de las palabras experimentales (sección 3.2) para poder estudiar las variables morfológicas en cuatro condiciones: 1) alta productividad-baja dificultad, 2) alta productividad-alta dificultad, 3) baja productividad-baja dificultad y 4) baja productividad-alta dificultad. Simultáneamente se controlaron las variables léxicas de cognado y frecuencia en la siguiente forma: i) palabras cognadas de alta frecuencia, ii) palabras cognadas de baja frecuencia, iii) palabras no cognadas de alta frecuencia y iv) palabras no cognadas de baja frecuencia. Asimismo, en la sección 3.3 explicaremos el diseño de las oraciones, las características de los participantes y la aplicación de la prueba. 


\subsection{Selección de sufijos experimentales}

Los sufijos para la prueba se eligieron a partir de la clasificación de Bauer y Nation (1993). De acuerdo a los criterios de estos autores, los sufijos aquí estudiados se distribuyen de la siguiente manera: dos sufijos pertenecen al nivel de dificultad 3 (-er y -able), dos al nivel 4 (-ation y -ment), uno al nivel $5(-a l)$ y uno al nivel $6(-y)^{6}$. Para esta investigación, los sufijos experimentales del nivel 4 se consideraron de baja dificultad porque tienen una sola función gramatical según el corpus Morphoquantics y además son cognados con el español (-(a)ción, y -miento); y los sufijos de los niveles 5 y 6 se consideraron de alta dificultad por tener diversas funciones gramaticales y no ser cognados con el español. La alta o baja productividad de los sufijos se calculó con la fórmula expuesta en Lowie (2005): $p=N \_$tipo / $N \_t o k e n$. En la tabla 1 se presentan los sufijos seleccionados y las cuatro condiciones experimentales.

\begin{tabular}{|l|c|c|}
\hline & Baja Dificultad (D-) & Alta Dificultad (D+) \\
\hline Alta Productividad (P+) & $\begin{array}{c}\text {-ation } \\
\text { (exploration) }\end{array}$ & $\begin{array}{c}- \text { al } \\
\text { (refusal) }\end{array}$ \\
\hline Baja Productividad (P- & $\begin{array}{c}-y \\
\text { (ment } \\
\text { (delivery) }\end{array}$ \\
\hline
\end{tabular}

Tabla 1. Sufijos experimentales seleccionados a partir de las variables morfológicas del estudio.

\subsection{Corpus y selección de palabras experimentales}

Las palabras fueron extraídas del corpus en línea de palabras complejas Morphoquantics, que contiene 17,943 tipos de palabras complejas derivadas de 835 morfemas derivacionales (554 prefijos y 281 sufijos). El corpus cuenta con 1,008,280 ocurrencias (tokens) recuperados de la parte oral del British National Corpus $(B N C)$ y nos ofrece la etimología y el significado de los morfemas, la categoría gramatical tanto de las bases a la que se añade el morfema como de las palabras derivadas que generan. También provee la clasificación del afijo (prefijo o sufijo), las diferentes palabras formadas con el morfema (types $=$ tipos de palabras) y el total de palabras en el corpus con el morfema en cuestión (tokens). Los morfemas en Morphoquantics están divididos en funciones gramaticales. Por ejemplo, podemos encontrar - ant ${ }_{1}$ como adjetivador deverbal (relieve -> relevant) y -ant ${ }_{2}$ como nominalizador deverbal (assist -> assistant). El número no indica que una función es más importante que otra pero el total de tipos de palabra podría sugerir las principales funciones de los morfemas. En este corpus, los sufijos -ation y -ment cuentan con la única función de nominalizadores deverbales, mientras que $-a l$ tiene dos funciones y el sufijo $-y$ tiene al menos siete ${ }^{7}$.

\footnotetext{
6 Los autores sugieren que los afijos más frecuentes, productivos, regulares y predecibles son los primeros en adquirirse y así los clasifican del nivel 1 (más fácil) al nivel 7 (más difícil). En este trabajo nada más se consideraron palabras terminadas con el sufijo -ation porque, a pesar de que puede considerarse como un alomorfo (-ation, -ition, -sion), los trabajos de Bauer y Nation (1993), Bauer et alii. (2013) y el corpus Morphoquantics lo analizan por separado. Considerar su alomorfia, cambiaría su nivel de dificultad y su productividad.

7 Algunas de las entradas del sufijo - $y$ en el corpus Morphoquantics no consideradas dentro de estas siete funciones se refieren a formas diminutivas, apreciativas o no (doggy, Daddy) e inclusive a interjecciones (oopsy!). http://
} 
En Morphoquantics, la lista de sustantivos deverbales que contienen el sufijo -ation es de 267 palabras, la de -ment de 194, la de -al de 37 palabras y la de - $y$ de 21 palabras. Una vez descargada la lista de sustantivos derivados con cada sufijo experimental, corroboramos su categoría gramatical y su valor semántico con varios diccionarios como el Online Etymology Dictionary, Merriam-Webster Dictionary y/o Cambridge English Dictionary. Esto nos permitió descartar sustantivos que tuvieran una base no verbal (butcher (n) -> butchery (n)) y que no tuvieran un significado de acción o resultado, eliminando así palabras derivadas con significado de objetos, lugares o personas.

De esta lista de palabras depurada, seleccionamos sustantivos de alta y baja frecuencia con cada sufijo experimental que fueran cognados y no cognados con el español. No se incluyeron falsos cognados. Para hacer la diferencia entre alta y baja frecuencia de manera objetiva y basada en el corpus, primero se eliminaron todas las que tuvieran una sola ocurrencia (hapaxes) para evitar derivados de tipo idiosincrático y posteriormente, con lo que restaba de la lista de palabras, se formaron deciles de frecuencia para los sufijos -ment y -ation y quintiles para los morfemas -al e $-y$. Para los sufijos -ment y -ation se consideraron de baja frecuencia las palabras que se encontraran entre los deciles 2 y 4 y de alta frecuencia las que se encontraran entre los deciles 7 y 9, eliminando así tanto los extremos como los deciles intermedios, siendo éstos últimos los que nos permiten hacer la división entre baja y alta frecuencia. La eliminación del decil 10 nos permitió descontar palabras cuyo gran número de ocurrencias puede deberse a la naturaleza del corpus.

La división en deciles tiene la ventaja de evitar decisiones arbitrarias. Por un lado, debido a que no hay un número igual de palabras con todos los sufijos experimentales, es imposible establecer un número de ocurrencias fijo que divida la baja y alta frecuencia; por otro lado, en caso de que se estableciera un número de ocurrencias distinto para cada sufijo experimental para hacer esta diferencia, dicha división sería poco confiable ya que se podrían obtener casos donde solo una ocurrencia marcara la diferencia entre baja y alta frecuencia. Así, en el caso del sufijo -ment, por ejemplo, se podría establecer un mínimo de 100 ocurrencias para considerar a una palabra de alta frecuencia pero si no se descartaran los deciles intermedios, correríamos el riesgo de tener una palabra de alta frecuencia con 100 ocurrencias y una de baja frecuencia con 99 ocurrencias, lo que sesgaría nuestros resultados.

Para los sufijos - al e - $y$ también se eliminaron los hapaxes pero debido al escaso número de palabras en el corpus, se tuvieron que considerar palabras de todos los quintiles de frecuencia, corroborando la división entre alta y baja frecuencia con la ocurrencia de las palabras en el $B N C$ en su sección escrita no académica (16.5 millones de palabras) y el Corpus of Contemporary American English (COCA) en su sección oral (118 millones de palabras $)^{8}$. El control del efecto de frecuencia de palabra y de la lengua materna resultó en cuatro condiciones léxicas: 1) palabras cognadas de alta frecuencia, 2) palabras no cognadas de alta frecuencia, 3) palabras cognadas de baja frecuencia y 4) palabras no cognadas

morphoquantics.co.uk/ También resaltamos que Bauer et.alii. (2013), basados en el corpus de inglés contemporáneo $C O C A$, señalan que el sufijo -ment es principalmente deverbal (development), pero también puede aparecer ocasionalmente añadido a adjetivos (oddment) u otros nombres (illusionment).

8 Estas cifras contrastan de manera importante con el millón de palabras que contiene en total el corpus Morphoquantics. El total de palabras del $B N C$ es de 100 millones de palabras y el de COCA es de más de 560 millones de palabras (consulta el 25 de mayo de 2018). 
de baja frecuencia. La prueba incluye cuatro palabras por cada condición léxica para cada sufijo experimental. Las listas de palabras experimentales se encuentran en la tabla 2.

\begin{tabular}{|c|c|c|c|c|}
\hline & $\begin{array}{l}\text { Bajo nivel de dificultad } \\
\text { y alta productividad } \\
\text { (-ation, } 16 \text { items) }\end{array}$ & $\begin{array}{l}\text { Alto nivel de dificultad } \\
\text { y baja productividad } \\
(-y, 16 \text { items })\end{array}$ & $\begin{array}{l}\text { Bajo nivel de dificultad } \\
\text { y baja productividad } \\
\text { (-ment, } 16 \text { items) }\end{array}$ & $\begin{array}{l}\text { Alto nivel de dificultad y } \\
\text { alta productividad } \\
\text { (-al, } 16 \text { items })\end{array}$ \\
\hline 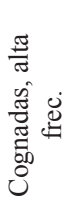 & $\begin{array}{l}\text { separation (32) } \\
\text { motivation (51) } \\
\text { collaboration (22) } \\
\text { integration (54) }\end{array}$ & $\begin{array}{l}\text { identity (61) } \\
\text { entry (146) } \\
\text { treaty (82) } \\
\text { subsidy (61) }\end{array}$ & $\begin{array}{l}\text { involvement (151) } \\
\text { treatment (322) } \\
\text { retirement (198) } \\
\text { requirement (258) }\end{array}$ & $\begin{array}{l}\text { disposal (63) } \\
\text { removal (86) } \\
\text { refusal (31) } \\
\text { approval (176) }\end{array}$ \\
\hline 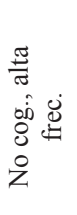 & $\begin{array}{l}\text { hesitation (23) } \\
\text { translation (40) } \\
\text { insulation (16) } \\
\text { taxation }(103)^{9}\end{array}$ & $\begin{array}{l}\text { recovery }(101) \\
\text { treasury }(80) \\
\text { delivery }(174) \\
\text { discovery }(54)\end{array}$ & $\begin{array}{l}\text { encouragement (68) } \\
\text { commitment (296) } \\
\text { improvement (178) } \\
\text { achievement (101) }\end{array}$ & $\begin{array}{c}\text { renewal (25) } \\
\text { arrival (34) } \\
\text { withdrawal (43) } \\
\text { dismissal (26) }\end{array}$ \\
\hline 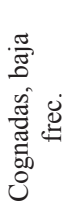 & $\begin{array}{l}\text { agitation (3) } \\
\text { vibration (2) } \\
\text { fascination (6) } \\
\text { devastation (6) }\end{array}$ & $\begin{array}{l}\text { robbery (34) } \\
\text { mastery (3) } \\
\text { fantasy (40) } \\
\text { continuity (35) }\end{array}$ & $\begin{array}{l}\text { accompaniment (4) } \\
\text { increment (6) } \\
\text { refinement (7) } \\
\text { confinement (5) }\end{array}$ & $\begin{array}{l}\text { dispersal (2) } \\
\text { reversal (9) } \\
\text { recital (3) } \\
\text { deferral (3) }\end{array}$ \\
\hline 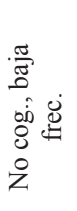 & $\begin{array}{c}\text { titration (1) } \\
\text { misappropriation (3) } \\
\text { exhilaration (4) } \\
\text { rumination (2) }\end{array}$ & $\begin{array}{l}\text { upholstery }(31)^{*} \\
\text { forgery }(26)^{*} \\
\text { flattery (4) } \\
\text { advocacy }(141)^{* 10}\end{array}$ & $\begin{array}{l}\text { adjournment (7) } \\
\text { nourishment (8) } \\
\text { astonishment (8) } \\
\text { amazement (6) }\end{array}$ & $\begin{array}{l}\text { retrieval (3) } \\
\text { burial (6) } \\
\text { portrayal (5) } \\
\text { betrayal (11) }\end{array}$ \\
\hline
\end{tabular}

Nota: El número entre paréntesis es el número de ocurrencias en el corpus Morphoquantics. Las palabras con asterisco no se encontraron en este corpus, por lo que su número de ocurrencias corresponde al $B N C$ escrito no académico, y cuyas palabras de alta frecuencia superan las 490 ocurrencias, a excepción de subsidy (186) y fantasy (181).

Tabla 2. Lista de palabras experimentales.

\subsection{Diseño y aplicación de la prueba experimental}

Se elaboró una prueba de producción controlada basada en la prueba de estructura morfológica de Carlisle (2000) que ha sido ampliamente usada con éxito tanto en estudios de la L1 (Muse, 2005; Stanfa, 2010) como en la L2 (Kieffer y Lesaux, 2008; Curinga,

9 A pesar de que en español existe la palabra tasación, ésta no se tomó como traducción de la palabra taxation ya que no significan lo mismo. Una tasación es una valoración (appraisal) mientras que taxation en el contexto de la prueba se refiere a una tributación o contribución, por lo que taxation se consideró una palabra no cognada en este estudio.

10 En inglés existen las palabras advocation (avocación en español, que se refiere a asistencia legal) y advocacy (acto o proceso de apoyar una causa). En este estudio se incluyó advocacy en la lista de palabras no cognadas porque en el contexto oracional nos interesa el significado de defensa o apoyo (no necesariamente legal). 
Ramírez et alii., 2011; 2013; Varatharajoo et alii., 2015) y se divide en dos tareas: una de descomposición (ver ejemplo 1) y otra de derivación (ver ejemplo 2). Nuestra prueba cuenta exclusivamente con la parte de derivación debido a que nuestro interés radica en la producción de los sufijos.

(1) (driver) Children are too young to drive.

(2) (farm) My uncle is a farmer.

Siguiendo a Sleeman y Brito (2010) con relación al continuo semántico eventivo $\rightarrow$ resultativo $\rightarrow$ objeto, y las propuestas de Peris y Delor (2009) y Mondoñedo (2011), consideramos que una oración tendría una lectura resultativa cuando contara con una o más de las siguientes características: i) la capacidad de pluralización del derivado, ii) que los determinantes que lo acompañen sean indefinidos, demostrativos o cuantificadores y iii) que el derivado aparezca con adjetivos relacionales (derivados generalmente de un sustantivo: intelecto $\rightarrow$ intelectual). Además, para garantizar el significado de la palabra derivada, se contó con la revisión de dos profesores de inglés, hablantes nativos de esta lengua. Construimos oraciones con una longitud máxima de diez palabras para evitar el cansancio de los participantes, y para inducir la respuesta dimos un verbo entre paréntesis al principio de la oración que los participantes debían derivar convirtiéndolo a sustantivo para completar correctamente la oración como en el ejemplo 3.

\section{(3) (betray) Accepting the money would be a betrayal of his principles.}

Colocamos la mitad de las palabras en posición pre-verbal y la otra mitad en posición postverbal con la finalidad de que los participantes leyeran las oraciones completas. En total, fueron 80 reactivos divididos en 64 reactivos de nominalización deverbal (16 para cada sufijo experimental); 16 reactivos distractores ( 8 para cada sufijo distractor) y 6 reactivos de familiarización (ejemplos). Los seis ejemplos fueron con los sufijos -y, -al, -ation, -ment, -ure y -ance, todos usados en su función de nominalizadores deverbales, siendo los cuatro primeros, sufijos experimentales. Para llegar a esta versión de la prueba se elaboraron y aplicaron dos pruebas piloto que se sometieron a una triple revisión: un experto en pruebas, un revisor de estadística y un hablante nativo del inglés que juzgó la naturalidad de las oraciones, el significado de las palabras en contexto y los contextos que aceptaban más de una respuesta correcta.

La modalidad de la aplicación de la prueba fue en línea usando una plataforma llamada Classmarker. Las instrucciones estaban en español e inmediatamente después se mostraban los ejemplos. La prueba comienza con siete preguntas de información personal (nombre, sexo, correo electrónico, rango de edad, nivel de proficiencia en inglés como L2, profesor y facultad) en orden fijo. Las siguientes 80 preguntas las recibían todos los participantes en un orden de presentación distinto y aparecían en sus pantallas una a la vez. No se les dio límite de tiempo para resolver la prueba y no se penalizaron errores ortográficos ni de concordancia de número. Las pruebas se aplicaron en el horario de la clase de inglés ya sea en el salón de clase de los participantes o bien en un laboratorio de cómputo y los profesores podían estar presentes. Al terminar la prueba, los participantes pudieron ver el porcentaje de respuestas correctas que obtuvieron y ver en qué se 
equivocaron, más no pudieron ver la respuesta correcta; igualmente, las investigadoras recibieron inmediatamente en su correo electrónico el porcentaje de respuestas correctas de cada participante, así como el tiempo que tardó cada uno en responder la prueba. Hubo un total de 130 participantes de una universidad pública de dos diferentes facultades, de los cuales 52 eran hombres y 78 eran mujeres. El nivel de inglés de los participantes estaba previamente establecido por la institución conforme al Marco Común Europeo de Referencia para las Lenguas. Para la investigación elegimos los grupos que pertenecieran a los niveles A2 $(\mathrm{N}=55), \mathrm{B} 1(\mathrm{~N}=46)$ y B2 $(\mathrm{N}=29)$.

\section{RESULTADOS: INCIDENCIA DE LAS CARACTERÍSTICAS MORFOLÓGI- CAS DE PRODUCTIVIDAD Y NIVEL DE DIFICULTAD EN EL APRENDIZAJE}

Como mencionamos anteriormente, esta investigación parte de la hipótesis de que los sufijos tienen características que facilitan su adquisición. En este caso, el sufijo experimental -ation cuenta con dos de estas características: ser de alta productividad y ser de baja dificultad, -ment tiene una característica facilitadora al ser de baja dificultad, -al también tiene solamente una al ser de alta productividad; y el sufijo - $y$ no tiene ninguna característica facilitadora para la adquisición. Basados en estas características, construimos las siguientes hipótesis experimentales:

H1. El sufijo de baja dificultad y alta productividad (-ation) obtendrá el mayor porcentaje de respuestas correctas en la prueba experimental.

H2. El sufijo de alta dificultad y baja productividad (-y) obtendrá el menor porcentaje de respuestas correctas en la prueba experimental.

En la tabla 3 podemos ver el porcentaje de aciertos de cada una de las cuatro condiciones morfológicas en los diferentes niveles de inglés como L2 y con el total de participantes. Cabe señalar que un análisis de varianza de medidas repetidas de la interacción "nivel de proficiencia de los participantes x productividad del sufijo x dificultad del sufijo" reveló que existen diferencias significativas entre niveles: $F(2,127)=46.72, p<0.001$, corroborado con análisis Post Hoc (Bonferroni).

\begin{tabular}{|c|c|c|c|c|}
\hline Nivel de L2 & $\begin{array}{c}\text { AltaProd/AltaDif } \\
(-a l)\end{array}$ & $\begin{array}{c}\text { AltaProd/BajaDif } \\
(- \text { ation })\end{array}$ & $\begin{array}{c}\text { BajaProd/BajaDif } \\
(-m e n t)\end{array}$ & $\begin{array}{c}\text { BajaProd/AltaDif } \\
(-y)\end{array}$ \\
\hline A2 & $5.34 \%$ & $49.20 \%$ & $17.50 \%$ & $15.11 \%$ \\
\hline B1 & $11.28 \%$ & $61.41 \%$ & $34.24 \%$ & $16.71 \%$ \\
\hline B2 & $24.57 \%$ & $75.86 \%$ & $54.09 \%$ & $32.97 \%$ \\
\hline Totales & $11.73 \%$ & $59.47 \%$ & $31.59 \%$ & $19.66 \%$ \\
\hline
\end{tabular}

Tabla 3. Porcentaje de aciertos de los morfemas experimentales en cada condición morfológica.

La tabla 3 muestra que la condición de alta productividad-baja dificultad, representada por el sufijo -ation obtiene los mejores resultados, apoyando así la hipótesis 1; sin embargo, 
la condición de baja productividad-alta dificultad, representada por el sufijo - $y$, no obtiene los resultados más bajos, rechazándose la hipótesis 2. Vemos que los resultados más bajos corresponden al sufijo - $a$ l, que tiene la propiedad facilitadora de ser de alta productividad y que el segundo sufijo con mejores resultados es -ment, que tiene como característica ser de baja dificultad, sugiriendo así que la baja dificultad facilita más el aprendizaje que la alta productividad.

Los resultados muestran que tanto la productividad como la dificultad tienen un efecto significativo (la productividad tiene una $F(1,127)=75.50, p<0.001$ y la dificultad una $F$ $(1,127)=543.97, p<0.001)$; i.e. estas dos características morfológicas facilitan la adquisición. Además encontramos un efecto significativo de la interacción del nivel de dificultad del sufijo con el nivel de proficiencia de los participantes $[F=(2,127) 10.23, p<0.001]$ al descubrir una mayor diferencia entre los aciertos de baja y alta dificultad en los niveles B1 y B2 en comparación con el nivel A2. Esto significa que los participantes más avanzados obtienen un mayor beneficio de la baja dificultad del sufijo. También encontramos un efecto significativo de la interacción de la productividad con la dificultad del sufijo $[F(1,127)=$ 253.36, $p<0.001]$. Como es esperado, los reactivos de baja dificultad tienen mejores resultados cuando la productividad es alta, (-ation obtiene mejores resultados que -ment) pero, inesperadamente, los reactivos de alta dificultad tienen mejores resultados cuando la productividad es baja ( $-y$ obtiene mejores resultados que $-a l$ ). Es posible que este último resultado no se pueda generalizar y se deba a otras características de estos dos sufijos no consideradas en esta investigación. No encontramos efectos significativos de la interacción de la productividad del sufijo con el nivel de proficiencia de los participantes ni de la interacción de la productividad del sufijo con su dificultad y el nivel de proficiencia de los participantes.

\section{RESULTADOS: INCIDENCIA LAS CARACTERÍSTICAS LÉXICAS DE FRE- CUENCIA Y COGNADO EN EL APRENDIZAJE}

Seguimos el mismo procedimiento de análisis para el nivel léxico. Primero enunciaremos las hipótesis experimentales, después veremos los porcentajes de aciertos en cada condición léxica y finalmente revelaremos los resultados del análisis estadístico. En el nivel léxico asumimos que las dos condiciones facilitadoras para la adquisición son i) ser palabras cognadas y ii) ser palabras de alta frecuencia. Por lo tanto tenemos palabras que cuentan con estas dos condiciones, palabras que cuentan con solo una de ellas y palabras que no cuentan con ninguna de ellas. Las hipótesis experimentales son:

H3. Los sustantivos deverbales cognados de alta frecuencia obtendrán el mayor porcentaje de respuestas correctas en la prueba experimental.

H4. Los sustantivos deverbales no cognados y de baja frecuencia obtendrán el menor porcentaje de respuestas correctas en la prueba experimental.

La tabla 4 muestra los resultados por niveles y con el total de los participantes en cada condición léxica experimental. En ella podemos apreciar que se aceptan ambas hipótesis, revelando así el efecto de las características facilitadoras de cognado y frecuencia. Un análisis de varianza de la interacción "nivel de proficiencia de los participantes x sustantivo cognado x sustantivo frecuente" arroja diferencias significativas entre niveles: F $(2,127)=47.37, \mathrm{p}<0.001$ 


\begin{tabular}{|c|c|c|c|c|}
\hline Nivel de L2 & $\begin{array}{c}\text { Cognado- } \\
\text { AltaFrec }\end{array}$ & $\begin{array}{c}\text { Cognado- } \\
\text { BajaFrec }\end{array}$ & $\begin{array}{c}\text { NoCognado- } \\
\text { AltaFrec }\end{array}$ & $\begin{array}{c}\text { NoCognado- } \\
\text { BajaFrec }\end{array}$ \\
\hline A2 & $28.27 \%$ & $24.73 \%$ & $22.09 \%$ & $12.27 \%$ \\
\hline B1 & $35.87 \%$ & $31.52 \%$ & $31.96 \%$ & $18.26 \%$ \\
\hline B2 & $55.17 \%$ & $44.48 \%$ & $51.55 \%$ & $31.03 \%$ \\
\hline Totales & $36.96 \%$ & $31.54 \%$ & $32.15 \%$ & $18.58 \%$ \\
\hline
\end{tabular}

Tabla 4. Porcentaje de aciertos en cada condición léxica.

La tabla 4 muestra que la condición léxica de cognado-alta frecuencia obtiene los mejores resultados, apoyando así la hipótesis 3 y la condición de no cognado-baja frecuencia obtiene los resultados más bajos, apoyando así la hipótesis 4 . Los porcentajes totales de la tabla 4 sugieren que las condiciones léxicas de cognado y de alta frecuencia inciden de manera muy similar en la adquisición. Las diferencias se encuentran entre los niveles A2 y B2. Mientras que los participantes del nivel A2 parecen beneficiarse más de la propiedad de cognado, en el nivel B2 parece que el hecho de que las palabras sean de alta frecuencia tiene mayor incidencia.

El análisis estadístico demuestra que ambas condiciones léxicas tienen un efecto significativo en la adquisición [cognado $\mathrm{F}(1,127)=113.55, \mathrm{p}<0.001$ y alta frecuencia $\mathrm{F}(1,127)$ $=245.04, \mathrm{p}<0.001)]$; es decir, una vez más, ambas condiciones facilitan la adquisición. El análisis también arroja efectos significativos de la interacción de frecuencia de palabra con el nivel de proficiencia de los participantes $[\mathrm{F}=(2,127) 14.21, \mathrm{p}<0.001]$ al mostrar que la diferencia de aciertos entre alta y baja frecuencia aumenta conforme sube el nivel de inglés de los participantes, sugiriendo que los estudiantes de mayor nivel de proficiencia se ven más beneficiados por la propiedad léxica de alta frecuencia. También existe un efecto de interacción de las propiedades léxicas de cognado y frecuencia $[\mathrm{F}(1,127)=31.08, \mathrm{p}<0.001]$ al encontrar que los cognados favorecen más a las palabras de baja frecuencia que a las de alta frecuencia; es decir, la diferencia de aciertos entre cognados y no cognados es mayor en las palabras de baja frecuencia. No se encontraron efectos significativos de la interacción de la propiedad de cognado con el nivel de proficiencia de los participantes ni de la interacción de propiedad de cognado con la propiedad de frecuencia y el nivel de proficiencia de los participantes.

\section{DISCUSIÓN Y CONCLUSIONES}

Los niveles propuestos por Bauer y Nation (1993) sirvieron como punto de partida en esta investigación al permitirnos elegir morfemas de diferente nivel de dificultad. Sin embargo, no sabíamos si estos niveles se mantendrían al ser controlados por significado y puestos a prueba empíricamente. Pudimos observar que -ation y -ment obtuvieron mayor porcentaje de respuestas correctas, sugiriendo que se adquieren antes que $-a l$ e $-y$ en acuerdo con lo que proponen los autores antes citados.

Nuestros hallazgos son similares a los reportados por Mochizuki y Aizawa (2000) en cuanto a que los sufijos -ation y -ment son de temprana adquisición y que las múltiples funciones de $-a l$ e $-y$ pueden ser parte de su complejidad. De hecho, ellos reportan que 
los participantes contestaron correctamente cuando - al se usaba como adjetivo, no como sustantivo. Esto puede explicar por qué en el efecto de interacción de "nivel de dificultad x productividad del morfema", el sufijo $-y$, a pesar de no contar con ninguna característica facilitadora para el aprendizaje, obtiene mejores resultados que -al. Es decir, hace falta estudiar estos sufijos con su función adjetivadora para contrastar los resultados.

Siguiendo en la misma línea, aunque Danilović et alii. (2013) no postularon un orden de adquisición para los sufijos, sus resultados muestran porcentajes de respuestas correctas que sugiere el siguiente orden de adquisición de los morfemas objeto de este estudio: -ation, -ment, -al e - $y$. Esto es interesante porque el estudio de Mochizuki y Aizawa (2000) fue con participantes japoneses y el de Danilović et alii. (2013) con participantes serbios, lo que sugiere que algunas características de los morfemas (como su multifunción) afectan el aprendizaje independientemente de la L1. También vale la pena señalar que a pesar de que nuestro estudio propone una metodología diferente a la de Mochizuki y Aizawa (2000) y Danilović et alii. (2013), llegamos a la misma conclusión referente a la adquisición. Mientras que ellos usaron un formato de opción múltiple con pseudopalabras y en los sufijos evaluaban la comprensión del conocimiento sintáctico, en este estudio se usó una tarea de producción controlada con palabras reales cognadas y no cognadas y de alta y baja frecuencia para evaluar el conocimiento distribucional. Lo enriquecedor de nuestro estudio es que empieza a discernir cuáles son algunas características que hacen un morfema de fácil o difícil adquisición, por lo menos para un tipo de población.

Aunque estamos conscientes de la diversidad de variables que se pueden controlar en una prueba experimental y lo importante que es hacerlo, consideramos que es difícil controlar demasiadas variables al mismo tiempo porque esto repercute en la selección de palabras. En esta investigación el corpus Morphoquantics fue muy útil por la información que ofrece sobre los morfemas y las palabras derivadas pero una de sus limitaciones es el escaso número de palabras que contiene con algunos sufijos. Otras variables no se pueden controlar por la naturaleza del objeto de estudio o porque se eligió una variable que incluye otras (pero no se puede concluir nada de las variables incluidas). Por ejemplo, en este estudio no se controló la longitud de palabra porque dos de los sufijos (-ation y -ment) tienen la facultad de crear palabras más largas que -al e $-y$, y al decidirnos por el nivel de dificultad del morfema, no profundizamos en cuestiones como neutralidad o multifunción del sufijo porque de cierto modo, ya estaba implicado en el nivel de dificultad. A pesar de que en este estudio solo se controlaron cuatro variables, resaltamos la necesidad de hacer más investigación con estas u otras variables para poder identificar su efecto en la adquisición morfológica porque reconocemos que este proceso es complejo y multifactorial.

Una de las limitaciones de este estudio es que, a juzgar por los resultados, la prueba fue complicada para los participantes de todos los niveles evaluados. Esto puede deberse a que: i) se trataba de una prueba de producción, que como ya mencionamos, suelen obtener resultados más bajos que las de comprensión, ii) que el conocimiento distribucional es en verdad el último en adquirirse y iii) a algunos casos de rivalidad morfológica ${ }^{11}$. Otra

11 De León (2010) estudia pares de sufijos que además de tener la misma función categorial, producen el mismo significado, proponiendo que sufijos como -ción y -miento entran en rivalidad, con casos de oponibilidad. Los autores explican que la rivalidad existe cuando dos derivaciones son posibles para la misma función categorial (ejem: predicamento y predicación) y que se presenta oponibilidad cuando el significado de las formas es diferente. Por ejemplo población se ha especializado en el sentido de "asentamiento humano" o "conjunto de habitantes" mientras 
limitación es que únicamente se evaluaron cuatro sufijos y dos de ellos son cognados con el español (-ation/-ación, -ment/-miento), lo cual nos da un efecto de cognados no sólo a nivel léxico sino también a nivel morfológico; y una limitación más es que no se evaluaron otros significados de los morfemas como objeto (equipment, illustration), lugar (basement) o persona(s) (army), lo cual nos podría indicar si el valor semántico también contribuye en la adquisición de los morfemas.

El estudio aporta algunas implicaciones pedagógicas. Por ejemplo, resalta el hecho de enseñar morfología. Así como hay autores que señalan la importancia de la instrucción en el reconocimiento de cognados (Bravo et alii., 2007 y Dressler et alii., 2011), este estudio apoya a los estudios que invitan a los maestros a enseñar morfología de manera explícita (Schmitt y Zimmerman, 2002; Kieffer y Lesaux, 2007; Khodadoust et alii., 2013) ya que, como sugieren Schmitt y Zimmerman (2002), "Los maestros no pueden asumir que los estudiantes van a absorber las formas derivativas automáticamente por la exposición [...] se necesita instrucción explícita de los sufijos". Esto se hace evidente con dos condiciones que dificultan de manera especial el aprendizaje de los sufijos: i) el hecho de que un sufijo puede tener varios significados y/o funciones, y 2) el hecho de que un solo significado puede expresarse por varios sufijos. El primer problema se ha abordado anteriormente por otros investigadores que sostienen que la función principal del morfema es la primera en adquirirse. Por ejemplo, en la escala de dificultad de Bauer y Nation (1993), -al como adjetivador (ejem: accidental) se encuentra en el nivel 4 mientras que -al como nominalizador (ejem: proposal) se encuentra en el 5, lo que sugiere que se adquiere más tardíamente ${ }^{12}, \mathrm{y}$ los datos del corpus Morphoquantics apoyan este planteamiento al mostrar 15 veces más tipos de palabras con la función adjetivadora de -al en comparación con su función nominalizadora. Pero un aspecto de naturaleza empírica que se ha dejado de lado es la coincidencia en significado de varios morfemas, como los sufijos aquí estudiados. Es necesario mostrar a los aprendices de inglés como L2 tanto la plurifuncionalidad de un sufijo como la variedad de sufijos que coinciden en su valor semántico. Esta enseñanza puede basarse en la productividad de las funciones de los sufijos y en los aspectos del conocimiento de la morfología derivativa. Por ejemplo: -er es un sufijo muy productivo para generar agentes (teacher) pero también existen los sufijos -ist (artist) y-ian (musician) que hacen lo mismo. Por otro lado, -er puede ser una forma comparativa como en bigger y además - er se añade a verbos (teach), mientras -ist y -ian se adhieren a sustantivos (art y music). En el primer caso, transformamos un verbo a un sustantivo mientras que en el segundo y tercer caso, se conservó la categoría gramatical de sustantivo pero con otro significado (ahora de agente). Este tipo de enseñanza puede darse poco a poco con análisis de palabras y palabras en contexto tomando en cuenta la exposición de los estudiantes a la L2 y dejando para niveles más avanzados los casos de rivalidad debidas a tecnicismos o sutilezas en el significado (advocacy vs advocation; dispersal vs dispersion) aunque existen casos de oponibilidad muy fuerte que probablemente se tengan que abordar antes (treatment vs treaty).

El presente estudio tenía como objetivo determinar cuál característica morfológica tiene mayor incidencia en el aprendizaje: el nivel de dificultad o la productividad. Se usaron cuatro sufijos que se distinguen en esas características pero que coinciden tanto en su fun-

que poblamiento se refiere a la actividad de poblar.

12 En el nivel 4 se encuentran afijos neutrales que se añaden a bases libres y el nivel 5, los afijos tienen estas mismas características pero son infrecuentes. 
ción gramatical como en su valor semántico (todos son nominalizadores deverbales con significado de resultado). También se analizaron efectos de facilitación a nivel léxico como las características de ser palabras cognadas o de alta frecuencia y se buscó determinar cuál de ellas incide más en el aprendizaje. Encontramos que las cuatro características tienen un efecto significativo en el aprendizaje. A nivel morfológico, identificamos que el bajo nivel de dificultad del morfema facilita más la adquisición que su alta productividad y a nivel léxico, que la alta frecuencia de las palabras beneficia más a los estudiantes avanzados mientras que la característica de cognado beneficia más a los principiantes, a pesar de que no hay efecto significativo estadístico entre estas últimas condiciones. Lo que sugiere es que los participantes principiantes, al tener menor exposición a la L2, dependen más de su L1. Estos resultados pueden ser útiles para maestros de inglés como L2 debido a que pueden guiar la secuencia de la enseñanza de morfemas; mientras que la metodología puede ser útil para otros investigadores interesados en estudiar el conocimiento distribucional de la morfología derivativa.

Nota: Nos gustaría agradecer a los revisores anónimos por sus observaciones a la versión anterior de este artículo para mejorarlo.

\section{Referencias bibliográficas}

Alotaibi, M. A. y Alotaibi, A. M. (2017). "On the Acquisition of Derivational Suffixes by Kuwaiti EFL Learners”. European Scientific Journal, ESJ, 13(17), pp. 223-238.

Bauer, L. (1983). English word-formation. Cambridge: Cambridge University Press.

Bauer, L., y Nation, P. (1993). "Word families", International journal of Lexicography, 6(4), pp. 253-279.

Bauer, L., Lieber, R. y Plag, I. (2013). The Oxford reference guide to English morphology. Oxford: Oxford University Press.

Bertram, R., Schreuder, R. y Baayen, R. H. (2000). "The balance of storage and computation in morphological processing: The role of word formation type, affixal homonymy, and productivity". Journal of Experimental Psychology: Learning, Memory, and Cognition, 26(2), pp. 489-511.

Bravo, M. A., Hiebert, E. H. y Pearson, P. D. (2007). "Tapping the Linguistic Resources of Spanish-English Bilinguals. The Role of Cognates in Science". En Wagner, R.K., A. Muse y K. Tannenbaum (eds.). Vocabulary acquisition: Implications for reading comprehension, Nueva York: Guilford, pp. 140-156.

Cambridge English Dictionary. Cambridge University Press. https://dictionary.cambridge.org/

Carlisle, J. F. (2000). "Awareness of the structure and meaning of morphologically complex words: Impact on reading", Reading and writing, 12(3), pp. 169-190.

Classmarker (2006). Sydney, Australia. https://www.classmarker.com/

Costa, A., Caramazza, A. y Sebastian-Galles, N. (2000). "The cognate facilitation effect: implications for models of lexical access". Journal of Experimental Psychology: Learning, Memory, and Cognition, 26(5), pp. 1283-1296.

Curinga, R. (2013). "Direct and Indirect Effects of Morphological Awareness on Reading Comprehension for Adolescent Spanish-English Emergent Bilinguals", BUCLD 37 Online Proceedings Supplement, pp. 1-12.

Danilović, J., Savić, J. D., y Dimitrijević, M. (2013). “Affix Acquisition Order in Serbian EFL Learners”, Romanian Journal of English Studies, 10(1), pp. 77-88.

De León, R. F. Z. P. (2010). "Esquemas rivales en la formación de palabras en español”. Onomázein: Revista de lingüistica, filología y traducción de la Pontificia Universidad Católica de Chile, (22), pp. 59-82. 
Dressler, C., Carlo, M. S., Snow, C. E., August, D. y White, C. E. (2011). 'Spanish-speaking students' use of cognate knowledge to infer the meaning of English words". Bilingualism: Language and cognition, 14(02), pp. 243-255.

Jiang, N., Novokshanova, E., Masuda, K. y Wang, X. (2011). "Morphological Congruency and the Acquisition of L2 Morphemes". Language Learning, 61(3), pp. 940-967.

Kelley, A. y Kohnert, K. (2012). "Is there a cognate advantage for typically developing Spanish-speaking English-language learners?” Language, Speech, and Hearing Services in Schools, 43(2), pp. 191-204.

Kieffer, M. J. y Lesaux, N. K. (2007). "Breaking down words to build meaning: Morphology, vocabulary, and reading comprehension in the urban classroom". The reading teacher, 61(2), pp. 134-144.

Kieffer, M. J. y Lesaux, N. K. (2008). "The role of derivational morphology in the reading comprehension of Spanish-speaking English language learners", Reading and Writing, 21(8), pp. 783-804.

Kieffer, M. J. y Lesaux, N. K. (2012). "Direct and indirect roles of morphological awareness in the English reading comprehension of native English, Spanish, Filipino, and Vietnamese speakers", Language Learning, 62(4), pp. 1170-1204.

Khodadoust, E., Aliasin, S. H. y Khosravi, R. (2013). "The relationship between morphological awareness and receptive vocabulary knowledge of Iranian EFL learners". International Journal of Educational Research and Technology, 4(1), pp. 60-67.

$\mathrm{Ku}$, Y. M. y Anderson, R. C. (2003). "Development of morphological awareness in Chinese and English". Reading and Writing, 16(5), pp. 399-422.

Lardiere, D. (2006). "Knowledge of derivational morphology in a second language idiolect". En Grantham, M., C. Shea y J. Archibald (eds.) Proceedings of the 8th generative approaches to second language acquisition conference (GASLA 2006). Massachusetts: Cascadilla Proceedings Project, pp. 72-79.

Lowie, W. (2000). "Cross-linguistic influence on morphology in the bilingual mental lexicon". Studia Linguistica, 54(2), pp. 175-185.

Lowie, W. (2005). "Exploring a second language: The discovery of morphological productivity", Eurosla yearbook, 5(1), pp. 251-268.

Marslen-Wilson, W., Tyler, L. K., Waksler, R. y Older, L. (1994). "Morphology and meaning in the English mental lexicon". Psychological review, 101(1), pp. 3-33.

Medellín Gómez, A. y Auza Benavides, A. (2008). "Influencia del tamaño del vocabulario en el conocimiento morfológico de afijos", Estudios de Lingüística Aplicada, 47, pp. 97-108.

Merriam-Webster Dictionary. Massachusetts, E.U. https://www.merriam-webster.com/

Mochizuki, M., y Aizawa, K. (2000). "An affix acquisition order for EFL learners: An exploratory study”. System, (28)2, pp. 291-304.

Mondoñedo, A. (2011). "Nominal participles, a case of categorical alternance: Eventive nominalization in -da", Anuario del Seminario de Filología Vasca "Julio de Urquijo", 39(2), pp. 161-174.

Morphoquantics. University of Reading. http://morphoquantics.co.uk (25/05/18).

Muse, A. E. (2005). The nature of morphological knowledge. (Tesis de doctorado). Florida State University. Florida, E.U.

Online Etymology Dictionary. Pensilvania, E.U. https://www.etymonline.com/

Peeters, D., Dijkstra, T. y Grainger, J. (2013). "The representation and processing of identical cognates by late bilinguals: RT and ERP effects". Journal of Memory and Language, 68(4), pp. 315-332.

Peris, A., y Delor, M. T. (2009). "Evaluación de los criterios lingüísticos para la distinción evento y resultado en los sustantivos deverbales", A survey of corpus-based research [Recurso electrónico], pp. 596-611.

Ramírez, G., Chen, X., Geva, E., y Kiefer, H. (2010). "Morphological awareness in Spanish-speaking English language learners: Within and cross-language effects on word reading", Reading and Writing, 23(3-4), pp. 337-358.

Ramírez, G., Chen, X., Geva, E., y Luo, Y. (2011). "Morphological awareness and word reading in English language learners: Evidence from Spanish-and Chinese-speaking children", Applied Psycholinguistics, 32(03), pp. 601-618. 
Schmitt, N., y Zimmerman, C. B. (2002). "Derivative word forms: What do learners know?" Tesol Quarterly, 36(2), pp. 145-171.

Sleeman, P. y Brito, A. M. (2010). "Nominalization, event, aspect and argument structure: A syntactic approach". En Duguine, M., S. Huidobro y N. Madariaga (eds.) Argument structure and syntactic relations: a cross-linguistic perspective. Philadelphia: John Benjamins Publishing, pp. 113-132.

Stanfa, K. (2010). Differentiating among students: The value added of a dynamic assessment of morphological problem-solving. (Tesis de doctorado). University of Pittsburgh. Pensilvania, E.U.

The British National Corpus, version 3. (2007). Oxford University Computing Services. http://www. natcorp.ox.ac.uk/ (25-05-18).

The Corpus of Contemporary American English. (2008). Brigham Young University. https:/corpus. byu.edu/coca/ (25-05-18).

Tyler, A., y Nagy, W. (1989). "The acquisition of English derivational morphology", Journal of memory and language, 28(6), pp. 649-667.

Varatharajoo, C., Asmawi, A. B., Abdallah, N., y Abedalaziz, M. (2015). "The Awareness of Morphemic Knowledge for Young Adults' Vocabulary Learning", The Malaysian Online Journal of Educational Science, 3 (2), pp. 45-56.

Ward, J., y Chuenjundaeng, J. (2009). "Suffix knowledge: Acquisition and applications", System, 37(3), pp. 461-469. 
